\title{
Fog in Comparison to Cloud: A Survey
}

\author{
K.P.Saharan \\ Department of Computer Engineering \\ Dnyanganga College of Engineering, Pune
}

\author{
Anuj Kumar \\ Department of Electronics and communication \\ Manav Rachna University, Faridabad
}

\begin{abstract}
Fog computing is new buzz word in computing world after cloud computing. This new computing paradigm could be seen as an extension to cloud computing. Main aim of fog computing is to reduce the burden on cloud by gathering workloads, services, applications and huge data to near network edge. In this survey paper, we will discuss main characteristics of the Fog that are; 1.Mobility, 2.Location awareness, 3.Low latency, 4.Huge number of nodes, 5. Extensive geographical distribution, 6.Various real time applications and we explore the advantages and motivation of Fog computing, and analyze its applications for IOT.
\end{abstract}

\section{Keywords}

Cloud, Fog, IOT, IOE,Wireless Networks.

\section{INTRODUCTION}

In last few years cloud computing gained huge popularity from research area to real time IOT applications. AnywhereAny time information access is possible only because of cloud computing.

As a whole Cloud computing provides various advantages to its user such as cost efficiency, Backup and Recovery, Easy Access to Information, Quick Deployment, Automatic Software Integration but there are some disadvantages also related with cloud like Technical Issues related with internet connection, Security issues, Limited control and flexibility, Dependency and vendor lock-in, Increased Vulnerability.

\subsection{Cloud computing}

There are two basic working models of cloud such as Service Models and Deployment Models.

\subsubsection{Service Models}

Service Models are the reference models on the basis of these models we can choose cloud provider. According to your need you can choose cloud services. These can be categorized into three basic service models as listed below:

Software as a Service (SaaS) : This model provide the facility to the user to pay for any application or service to access anywhere any time from the cloud. This model uses third party vendor. All applications are delivered through and managed by a third-party vendor and whose interface is access on the user side

Platform as a Service (PaaS) : This model provide the facility to the user to pay for the access to the platforms, allow them to deploy their own applications and software in the cloud.

Infrastructure as a Service (IaaS) : This model provide the facility to the user to manage and control the systems in terms of the applications, operating systems, network connectivity and storage but there is no need to control the cloud infrastructure.

There are various other service models like XaaS, i.e., anything as a Service. This can be Business as a Service, Database as a Service, Network as a Service, Strategy as a Service or Identity as a Service.

\subsubsection{Deployment Models}

The deployment models have been identified according to needs of the services and users of the clouds in specific ways with the different characteristics of the four different models.

Private Cloud: This cloud infrastructure has been deployed, operated and is maintained by a particular organization and not shared with other organizations.

Community Cloud: This cloud infrastructure is shared with a number of organizations with similar requirements and interests.

Public Cloud: This cloud infrastructure is available to the public on a commercial basis by a cloud service provider. This model provides an on-demand solution Payment is made on a per use basis.

Hybrid Cloud: This cloud infrastructure is combination of clouds of any type. This can be a combination of public and private clouds or public or community cloud according to the need of services and applications required by the end user.

\section{LIMITATIONS AND CHALLENGES OF CLOUD COMPUTING}

In comparison to the previous traditional computing paradigms, Cloud computing provides various advantages to both service providers and users but it has also some limitations and challenges.

Security and Privacy: Data security is major issues related with personal data and confidential data of organizations. User has to completely depend upon the cloud service provider for their data privacy and security.

Technical Issues: High speed internet connectivity requirement makes the system complex. Various technical issues arise during high load.

Data lock-in: The lack of standard APIs restricts the migration of applications and services between clouds. With the rise of cloud the problems of Data portability, migration and vendor lock-in situation will increase.

Data segregation: Mostly Data segregation problem arises in the multi-tenant usage mode, where the different users' virtual machines are co-located on the same hard disks or same server. Here the risks includes to properly separate storage or memory between different users.

Data location: The geographic location of the data is also very important to secure the data and information of client. Rules and regulation for certain types of data is different in the different countries. A customer could be involved in illegal issues without even noticing. 
Recovery and back-up: Data protection and recovery is an important aspect of cloud .some times in disaster situations recovery process is quite slow.

\section{FOG COMPUTING}

Cisco system introduced a new term "fog computing " to make the data transfer easy in wireless and distributed environment[7].According to Bonomi et al., [7] the rationale for coining this term to identify this model is that fog is nothing but cloud that is closer to the ground. Hence cloud computing carried out closer to the end users' networks is thus identified as fog computing. Fog computing is a creation of virtualized platform that is located between cloud data centers hosted within the Internet and end user devices [7]. fog computing can provide better QOS in terms of, Energy consumption, delay and reduced data traffic over the internet etc [5].

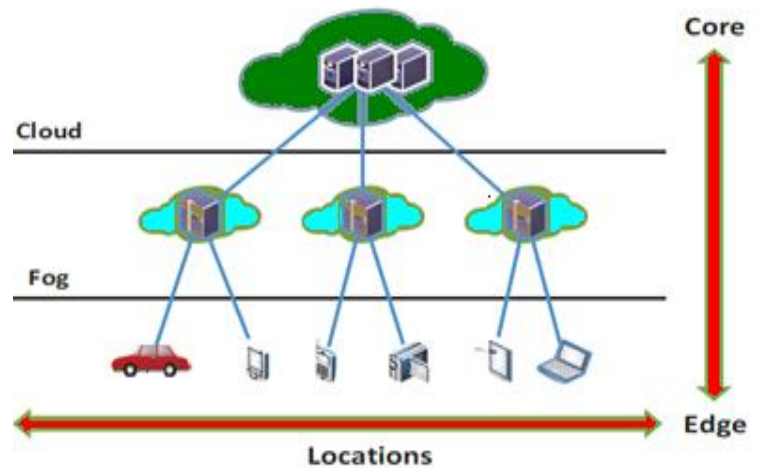

Figure: 1 Cloud and Fog comparison on Edge [3]

The key advantages of Fog Computing are related to:

Keeping the Data close to the user: To eliminate the delays in data transfer fog allows keeping the data close to the user instead of store them in a far datacenters.

Dense geographical distribution : Fog computing creates an edge networks which sits at various points to extends the direct cloud services dense, geographically isolated infrastructure helps to handle and analyze big data faster, traversal to the entire WAN can be restricted because the administrators are able to support location-based mobility demands .

Great support for mobility: There is a tremendous increase in the amount of data and devices. A fog system supports to handle this large data and information and provides a better and faster way to access and analyze the data.

Save storage space: Fog computing would be a great option to prevent inappropriate or irrelative information to travel to the overall network; this will save storage space and reduces the delay.

Support for IOT: Fog computing scenario can be applied in a number of areas where Internet of Things is present. It may plays an important role in various application in IOT Like- in the working of Smart Traffic Lights and Vehicles, smart grids, smart cities, Wireless Sensor network and Actuator and Cyber-physical systems .

Extension of cloud and integration with other services : Fog computing could not be considered as the replacement of the cloud, this is a kind of extension to provide filtered and faster inputs to the cloud and users.

\section{APPLICATIONS AREA OF FOG}

Wireless Sensor and Actuator Networks: In previous wireless sensor networks various applications are difficult for sensing and tracking because of their complex physical actions. For this, in this scenario, actuators allocated as Fog devices can control the measurement process itself, oscillatory behaviors and the stability by creating a closed-loop system.[3]

Smart Grid: Network edge devices, such as micro-grids and smart meters works for Energy load balancing applications. Based on availability[3], energy demand and the lowest price, these kind of devices without human intervention switch to alternative energies like wind and solar.

Smart Traffic Lights and Connected Vehicles: The presence of pedestrians and bikers can be detected by the smart street light to interact locally with sensors and they can measure the Nearby smart lights serving as Fog devices coordinate to create green traffic wave and send warning signals to approaching vehicles [2]

Fog and IOT: IOT is a kind of network that can be considered as interconnection of the ordinary physical or daily life object to the technology. Fog computing helps the concept of IoT by using edge networking.

\section{COMPARISON OF CLOUD AND FOG COMPUTING}

The concept of Fog computing is very much similar to cloud computing. But following few parameters shows the difference between these two close concepts. Table 1 summarizes comparison between cloud and fog computing. [2]

Table 1. Comparisons of cloud and Fog on different parameters

\begin{tabular}{|l|l|l|}
\hline Parameters & $\begin{array}{l}\text { Cloud } \\
\text { Computing }\end{array}$ & Fog Computing \\
\hline $\begin{array}{l}\text { Server nodes } \\
\text { location }\end{array}$ & $\begin{array}{l}\text { Within the } \\
\text { Internet }\end{array}$ & $\begin{array}{l}\text { At the edge of the } \\
\text { local network }\end{array}$ \\
\hline $\begin{array}{l}\text { client and } \\
\text { server distance }\end{array}$ & Multiple hops & Single hop \\
\hline Latency & High & Low \\
\hline Delay Jitter & High & Very low \\
\hline Security & $\begin{array}{l}\text { Less secure, } \\
\text { Undefined }\end{array}$ & $\begin{array}{l}\text { More secure, Can be } \\
\text { defined }\end{array}$ \\
\hline $\begin{array}{l}\text { Awareness } \\
\text { about location }\end{array}$ & No & Yes \\
\hline vulnerability & High probability & Very low probability \\
\hline $\begin{array}{l}\text { Geographical } \\
\text { distribution }\end{array}$ & Centralized & $\begin{array}{l}\text { Dense and } \\
\text { Distributed }\end{array}$ \\
\hline $\begin{array}{l}\text { Number of } \\
\text { server nodes }\end{array}$ & Few & Very large \\
\hline $\begin{array}{l}\text { Real time } \\
\text { interactions }\end{array}$ & Supported & Supported \\
\hline $\begin{array}{l}\text { kind of last } \\
\text { mile } \\
\text { connectivity }\end{array}$ & Leased line & Wireless \\
\hline Mobility & Limited support & Supported \\
\hline
\end{tabular}

\section{CONCLUSIONS}

On the basis of above survey, we can conclude that Fog computing is extension of cloud with some extra features for 
service provider and end user. Fog Computing is not a replacement for Cloud Computing. In this paper, we explain cloud computing model and limitations of cloud computing that is probable reason for the birth of fog computing. According to the present scenario of huge data, IOT and IOE fog computing performs better than cloud computing. Till now fog computing is working as a support system to the present cloud computing systems. In future Edge concept in fog will give new opportunities and solutions to network operators and end users. Fog computing will provide better quality of service to the applications like smart grid and connected vehicles and will give new service models in future. Thus fog computing adds one more feather to IOTs cap.

\section{REFERENCES}

[1] Manreet kaur, Monika Bharti “ Fog Computing Providing Data Security: A Review",in International Journal of Advanced Research in Computer Science and Software Engineering, Volume 4, Issue 6, June 2014

[2] Mohamed Firdhous, Osman Ghazali and Suhaidi Hassan " Fog Computing: Will it be the Future of Cloud Computing?,in Proceedings of the Third International Conference on Informatics \& Applications, Kuala Terengganu, Malaysia, 2014

[3] Ivan Stojmenovic, Sheng Wen," The Fog Computing Paradigm: Scenarios and Security Issues, Proceedings of the 2014 Federated Conference on Computer Science and Information Systems pp. 1-8

[4] F.Bonomi, R.Milito, J.Zhu, and S.Addepalli, "Fog computing and its role in the Internet of Things," in ACM SIGCOMM Workshop on Mobile cloud Computing, Helsinki, Finland, 2012, pp. 13--16.

[5] D.Kovachev, "Mobile multimedia services in the cloud," Ph.D. dissertation, RWTH Aachen University, Aachen, Germany, 2014.
[6] J.K. Zao, T.T. Gan, C.K. You, C.E. Chung, Y.T. Wang, S.J.R. Mendez, T.Mullen, C.Yu, C.Kothe, C.T. Hsiao, S.L. Chu, C.K. Shieh, and T.P. Jung, "Pervasive brain monitoring and data sharing based on multi-tier distributed computing and linked data technology," Frontiers in Human Neuroscience, vol.8, no. 370, pp. 1-$16,2014$.

[7] Archer, Jerry, et al. "Top threats to cloud computing v1. 0." Cloud Security Alliance (2010).

[8] F. Bonomi, "Connected vehicles, the internet of things, and fog computing,"in The Eighth ACM International Workshop on Vehicular Inter-Networking (VANET), Las Vegas, USA, 2011.

[9] Cisco, "Cisco delivers vision of fog computing to accelerate value frombillions of connected devices," Cisco, Tech. Rep., Jan. 2014.

[10] K. Hong, D. Lillethun, U. Ramachandran, B. Ottenwälder, and B. Koldehofe,"Opportunistic spatiotemporal event processing for mobile situationawareness," in Proceedings of the 7th ACM International Conferenceon Distributed Event-based Systems, ser. DEBS'13. ACM, 2013,pp. 195-206.

[11] H. Madsen, G. Albeanu, B. Burtschy, and F. PopentiuVladicescu,"Reliability in the utility computing era: Towards reliable fog computing,"in Systems, Signals and Image Processing (IWSSIP), 2013 20thInternational Conference on, July 2013, pp. 43-46.

[12] K. Hong, D. Lillethun, U. Ramachandran, B. Ottenwälder, and B. Koldehofe,"Mobile fog: A programming model for large-scale applications on the internet of things," in Proceedings of the Second ACM SIGCOMM Workshop on Mobile Cloud Computing, ser. MCC'13. ACM, 2013, pp.15-20. 